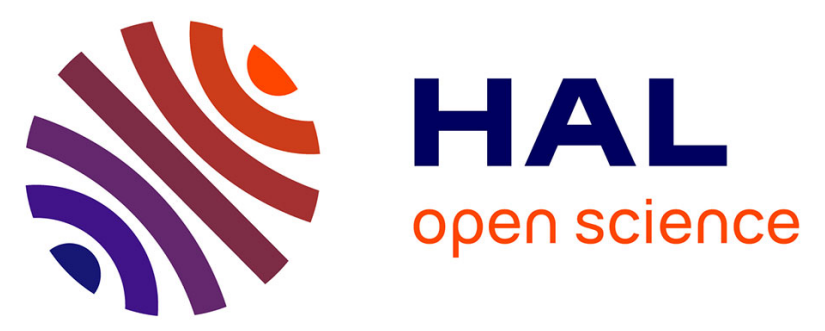

\title{
Dose point kernels in liquid water: An intra-comparison between GEANT4-DNA and a variety of Monte Carlo codes
}

Christophe Champion, Sébastien Incerti, Yann Perrot, Rachel Delorme, Marie-Claude Bordage, Manuel Bardiès, Barbara Mascialino, Ngoc-Hoang Tran, Vladimir Ivanchenko, Mario Bernal, et al.

\section{To cite this version:}

Christophe Champion, Sébastien Incerti, Yann Perrot, Rachel Delorme, Marie-Claude Bordage, et al.. Dose point kernels in liquid water: An intra-comparison between GEANT4-DNA and a variety of Monte Carlo codes. Applied Radiation and Isotopes, 2014, 83 (Part B), pp.137-141. 10.1016/j.apradiso.2013.01.037 . hal-00858479

\section{HAL Id: hal-00858479 https://hal.science/hal-00858479}

Submitted on 6 Sep 2013

HAL is a multi-disciplinary open access archive for the deposit and dissemination of scientific research documents, whether they are published or not. The documents may come from teaching and research institutions in France or abroad, or from public or private research centers.
L'archive ouverte pluridisciplinaire HAL, est destinée au dépôt et à la diffusion de documents scientifiques de niveau recherche, publiés ou non, émanant des établissements d'enseignement et de recherche français ou étrangers, des laboratoires publics ou privés. 

an intra-comparison between GEANT4-DNA and a variety of Monte Carlo codes

${ }^{\mathrm{b}}$ Laboratoire de Physique Corpusculaire, Université Blaise Pascal, CNRS/IN2P3, Aubière, France.

${ }^{\mathrm{c}}$ CEA, LIST, Laboratoire Modélisation, Simulation et Systèmes, Gif-sur-Yvette, France.

10 Laboratoire Plasmas et Conversion d'énergie, Université Paul Sabatier, Toulouse, France.

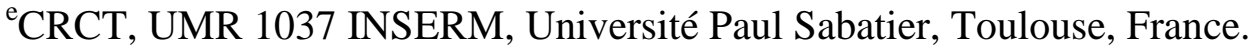

${ }^{\mathrm{h}}$ Instituto de Física Gleb Wataghin, Universidade Estadual de Campinas, SP, Brazil.

\section{Abstract}

21 Modelling the radio-induced effects in biological medium requires accurate physics models to

22 describe in detail the main physical interactions induced by all the charged particles present in 23 the irradiated medium (secondary as well as primary ones). These interactions include

24 inelastic events like ionization and excitation processes as well as elastic scattering, the latter

25 being the most important process in the low-energy regime. To check the accuracy of the 
26 theoretical models recently implemented into the Geant4 toolkit for modelling the electron

27 slowing-down in liquid water, the simulation of electron Dose Point Kernels remains the

28 preferential test. In this work, normalized radial profiles of deposited energy at a distance

29 from emissions point sources are then computed in liquid water by using the very low energy

30 "Geant4-DNA" physics processes available in the Geant4 toolkit. We here report an extensive

31 comparison with profiles obtained by a large selection of existing and well-documented

32 Monte-Carlo codes, namely, EGSnrc, PENELOPE, CPA100, FLUKA and MCNPX.

34 Keywords: Dose Point Kernel; Geant4-DNA; Monte Carlo codes; liquid water.

37 PACS: 87.53.Bn; 02.70.Ss; 87.50.-a; 87.53.-j

\section{Corresponding author}

41 Christophe Champion

42 Université Bordeaux 1, CNRS/IN2P3, Centre d'Etudes Nucléaires de Bordeaux Gradignan,

43 CENBG, Chemin du Solarium, BP120, 33175 Gradignan, France

44 Tel: +33-5-57-12-08-89; fax: +33-5-57-12-08-01

45 E-mail address: champion@ @enbg.in2p3.fr

46 


\section{1. Introduction}

48 Energy deposition functions from point isotropic sources - commonly denoted dose point

49 kernel (DPK) functions - are of prime interest in many fields like dosimetry in particular for

50 medical applications. To better understand the radiobiological effects resulting from the use

51 of electron-emitting radiopharmaceuticals, it is necessary to have an appropriate knowledge of

52 the cellular distribution of the radiopharmaceutical and then to model the microscopic

53 distribution of energy deposited in irradiated matter [1]. Absorbed doses to targeted cancer

54 cells play an important role in evaluating the relative merits of different radionuclides and

55 pharmaceuticals. In this context, information on the bio-distribution at the tissue, cellular and

56 sub-cellular levels can be obtained by autoradiography [2], micro-autoradiography [3], or

57 alternative techniques such as secondary ion mass spectrometry [4]. Converting these data to

58 absorbed dose distribution requires the use of analytic methods based on point-dose kernels or

59 methods based on radiation transport calculations [5-7]. Indeed, Monte Carlo code event-by-

60 event simulations can be particularly suitable [7-11]. The latter consist in describing, step-by-

61 step, interaction after interaction, the history of each ionizing particle created during the

62 irradiation of the biological matter. In this kind of numerical code, each projectile-target

63 interaction is described either thanks to theoretical (differential as well as total) cross sections

64 or by semi-empirical ones giving access to a more or less complete description of the

65 kinematics before and after the collision.

66 In fact, there are in the literature a large number of Monte Carlo electron track-structure

67 codes in water, which have been developed independently to investigate the microscopic

68 features of ionizing radiation, the ensuing chemical pathways and the molecular nature of the

69 damages in bio-molecular targets (see [11] and references therein). The aim of the present

70 study is to compare dose point kernels - for particular electron energies - calculated by using

71 different Monte Carlo codes, namely, EGSnrc [12], PENELOPE [13], CPA100 [14], FLUKA 
72 [15], MCNPX [16] and GEANT4-DNA [17]. To do that, the energy deposited by the emitted

73 electrons as well as all the secondary particles produced along the primary trajectories are

74 scored in spherical shells placed around an isotropic source for distances ranging from 0 to

751.2 times the continuous slowing-down approximation range hereafter denoted $R_{C S D A}$ and

76 provided by the different codes here studied.

\section{2. Methods}

79 The Monte Carlo numerical simulations used in the present study are well-documented and

80 nowadays extensively used by many groups. Only a brief description is then hereafter

81 reported and for more details we refer the interested reader to the corresponding literature

82 whose examples are cited as references.

\subsection{The GEANT4-DNA code}

84 The Geant4-DNA code is fully included in the general purpose Geant4 Monte Carlo

85 simulation toolkit. It simulates track structures of electrons, hydrogen and helium atoms of 86 different charge states $\left(\mathrm{H}^{0}, \mathrm{H}^{+}\right)$and $\left(\mathrm{He}^{0}, \mathrm{He}^{+}, \mathrm{He}^{2+}\right)$ respectively, as well as $\mathrm{C}^{6+}, \mathrm{N}^{7+}, \mathrm{O}^{8+}$ and $87 \mathrm{Fe}^{26+}$ ions, in liquid water. The physical processes include ionization (for all particles), 88 electronic excitation (for electrons, protons, hydrogen atoms and $\alpha$-particles including their 89 different charge states), charge exchange (for hydrogen and helium atoms with the above90 mentioned charge states), and, for electrons, elastic scattering, vibrational excitation and 91 dissociative attachment. Electron interactions cover the $7.4 \mathrm{eV}-1 \mathrm{MeV}$ energy range, whereas

92 proton and hydrogen interactions are simulated from $100 \mathrm{eV}$ to $100 \mathrm{MeV}$ while helium ions of 93 different charged states are followed from $1 \mathrm{keV}$ up to $400 \mathrm{MeV}$. These processes are further 94 described in [17].

95 2.2 The EGSnrc code 
96 EGSnrc is a general-purpose package for the Monte Carlo simulation of the photons and the

97 electrons transport from a few $\mathrm{keV}$ up to $100 \mathrm{GeV}$. EGSnrc uses a condensed history approach

98 based on the formalism developed by Kawrakow and Bielajew to sample angular distributions

99 from the any-angle form of the screened Rutherford cross section [18]. The Möller inelastic

100 cross-sections are used for the generation of secondary electrons. For this study, the

101 simulations were based on the user-code EDKnrc developed by Mainegra et al. [19]. We

102 applied the PRESTA II electron-step algorithm and the EXACT boundary crossing algorithm

103 to switch to single scattering when a particle comes closer to a boundary. The "skin depth"

104 parameter was set to 3: it represents the number of elastic mean free paths to the next

105 boundary at which the simulation switches into single scattering mode. We set the cut-off

106 parameter ECUT to $1 \mathrm{keV}$ in order to track primaries and secondaries until they leave the

107 geometry or their energy falls below $1 \mathrm{keV}$. We produced a PEGS4 data set describing cross

108 sections and stopping powers adapted for this low cut-off value.

1092.3 The PENELOPE code

110 PENELOPE (2006 version) is a general-purpose Monte Carlo code for the coupled simulation

111 of electron and photon transport. The cross sections database used in PENELOPE covers a

112 wide range of elements $(Z=1-99)$ and various materials useful for medical applications in the

113 energy range of $50 \mathrm{eV}-1 \mathrm{GeV}$. This code has the flexibility to generate electron and positron

114 histories on the basis of a mixed procedure, which combines detailed simulation of hard

115 events with the continuous slowing down approximation for soft interactions. The level of

116 detail of electron transport processes is controlled in PENELOPE by specifying values for

117 several parameters, $C_{1}, C_{2}, W_{C C}$ and $W_{C R}$. The $C_{1}$ and $C_{2}$ parameters are associated to the 118 condensation of electron and positron elastic scattering processes. $W_{C C}$ and $W_{C R}$, respectively, 119 represent the cut-off energy losses for hard inelastic collisions and for hard Bremsstrahlung 120 emission. A detailed description of the algorithms used in PENELOPE can be found in its 
121 manual [20]. These simulations were done with detailed event-by-event transport setting

$122 C_{1}=C_{2}=0, W_{C C}=W_{C R}=50 \mathrm{eV}$ and using $50 \mathrm{eV}$ as the lower absorption energy allowed in

123 this code.

1242.4 The CPA100 code

125 CPA100 is an event by event Monte Carlo track structure code, developed in Toulouse

126 (France), for understanding fundamental aspects of radiation track interaction [14]. It

127 simulates complete electron/photon transport in liquid water for energy range from 10 to

$128200 \mathrm{keV}$. It generates all the electronic and photonic cascades occurring after a particle

129 passage in the volume of interest (Auger electron, X-Rays, atomic reorganization). It is also

130 able to describe the various stages of the particle transport not only the early physical stage,

131 but also the physico-chemical and the chemical ones, during the very early passage of

132 particles in matter say up to one microsecond. Primary physical and chemical damages not

133 only in liquid water but also in complex DNA targets and its higher order structures can be

134 calculated to estimate the radio-induced damage to the DNA molecular scale (DSB, SSB,

135 base lesion).

136 2.5 The FLUKA code

137 FLUKA is a multi-purpose Monte Carlo particle transport code that considers all particle

138 interactions including electromagnetic interactions, nuclear interactions of the primary or

139 incident particles and the generated secondary particles, energy loss fluctuations and Coulomb

140 scattering [15]. The version 2011.2.15 with the default configuration 'PRECISION' was used,

141 with an energy cut-off lowered at $1 \mathrm{keV}$ for electrons and $0.1 \mathrm{keV}$ for photons. To reach a

142 good accuracy, the single scattering model through the 'MULSOPT' option was activated,

143 because the Moliere multiple scattering model could be unreliable with thin shells, disturbing

144 the propagation of electrons between the boundaries [21].

145 2.6. The MCNPX code 
146 MCNPX is a general-purpose Monte Carlo code for modelling the interaction of radiation

147 with matter [16]. MCNPX stands for MCNP eXtended and transports electrons, photons,

148 neutrons and several particle types, like nearly all energies. It utilizes the latest nuclear cross

149 section libraries and covers various materials useful for medical applications. The tallies have

150 extensive statistical analysis and the convergence is enabled by a wide variety of variance

151 reduction methods. For this work, the version 2.7 .0 was used with the F8* energy deposition

152 tally in coupled electron-photon mode. The photon and electron cut-off energies were set

153 above $1 \mathrm{keV}$. A specific consideration was focused on electron transport conditions, through

154 the ITS option and the ESTEP parameter, due to the very narrow shells. The ITS energy

155 indexing algorithm was used to have a better definition of the energy group and their

156 boundaries [22] and the ESTEP parameter was increased in order to divide the major electron

157 energy step into smaller sub-steps [23]: ESTEP = 10 for $100 \mathrm{keV}$ and ESTEP $=100$ for 10, 30

158 and $50 \mathrm{keV}$.

\section{Results and discussion}

161 To obtain the dose point kernel (DPK) around an isotropic point source, the geometry

162 here used consists in a spherical water phantom divided into 120 spherical shells of thickness

$163 R_{C S D A} / 100$, where $R_{C S D A}$ stands for the continuous-slowing-down-approximation range whose

164 values calculated by the different codes here studied are reported in Table 1 . Note that for the

165 EGSnrc, the CPA100, the FLUKA and the MCNPX codes, the corresponding values are taken

166 from the NIST web database ESTAR [24], what generates stopping powers and ranges for

167 electrons which are the same as those tabulated in ICRU Report 37 [25]. Besides, let us 168 remind that the present GEANT4-DNA version transports electrons down to an energy 169 threshold of $7.4 \mathrm{eV}$ contrary to the other codes studied which use higher energy cut-off, what 170 undoubtedly affects the $R_{C S D A}$ values. 
Finally, the GEANT4-DNA DPK distributions have been compared to those obtained

172 with the other Monte Carlo codes by using Kolmogorov-Smirnov statistical tests. Thus, we

173 found that the GEANT4-DNA simulations are statistically compatible with EGSnrc and

174 PENELOPE simulations ( $p$-value $>0.05$ ) with a maximum distance $(D)$ between distribution

175 functions less than 0.2 . On the contrary, much smaller $p$-values $(<0.05)$ and larger $D$

176 distances were obtained when comparing GEANT4-DNA simulations with the MCNPX and

177 CPA100 simulations.

178 The DPK distributions also obtained by the different numerical codes are reported in

179 Figure 1 for four particular electron energies, namely, $10 \mathrm{keV}, 30 \mathrm{keV}, 50 \mathrm{keV}$ and $100 \mathrm{keV}$.

180 These quantities are defined as the fraction of the emitted energy absorbed (per unit mass) at a

181 certain distance from the point source and are usually reported by means of scaled

182 distributions defined as $F\left(r / R_{C S D A}\right)=\frac{\delta E(r) / E_{0}}{\delta r / R_{C S D A}}$ where $r$ is the distance from the point source,

$183 \delta E(r)$ stands for the energy absorbed in the spherical shell sited at a distance $r$ from the point

$184 r / R_{C S D A}$ source, $E_{0}$ being the initial kinetic energy of the electron and $\delta r$ the shell thickness

185 (here $R_{C S D A} / 100$ ). The obtained distributions will be hereafter reported as a function of $r / R_{C S D A}$

186 and refer to scoring of the deposited energy at the mid-radius of the shell.

187 In Figure 1, we observe that the shape of the dose point kernels generated by the 188 different codes is very similar. However, we note that the CPA100 code exhibits a peak closer 189 to the source in comparison to the other codes $\left(r / R_{C S D A} \cong 0.53\right.$ vs 0.58$)$, the amplitudes being 190 all of the same order of magnitude - from 1.45 to 1.55 - except for the MCNPX which largely 191 overestimates the other results. When the incident electron energy increases, these 192 observations are confirmed with in particular an improvement of the agreement between the 193 CPA100 and the other simulations. Thus, from Fig.1b) to Fig.1d) all the curves tend to 194 converge except again the MCNPX simulation which provides higher DPKs (of about 20\%). 
Besides, for the four energetic cases here reported, the GEANT4-DNA DPK

196 distributions have been compared to those obtained with the other Monte Carlo codes by

197 using Kolmogorov-Smirnov statistical tests. Thus, we found that the GEANT4-DNA

198 simulations are statistically compatible with EGSnrc, PENELOPE and FLUKA simulations

199 ( $p$-value $>0.05)$ with a maximum distance $(D)$ between distribution functions less than 0.3.

200 On the contrary, much smaller $p$-values $(<0.05)$ and larger $D$ distances were obtained when

201 comparing GEANT4-DNA simulations with the MCNPX (for the four incident energy

202 values) and CPA100 (for $30 \mathrm{keV}$ and $50 \mathrm{keV}$ ) simulations.

203

204 4. Conclusions

205 Normalized radial profiles of deposited energy - commonly referred to as dose point kernels -

206 have been here reported by using the very low energy "Geant4-DNA" physics processes

207 available in the Geant4 toolkit. In comparison with profiles obtained by a large selection of

208 existing and well-documented Monte-Carlo codes, namely, EGSnrc, PENELOPE, CPA100,

209 FLUKA and MCNPX, we have here emphasized evident discrepancies undoubtedly relied to

210 the physics models implemented into the different codes. In this context, the Geant4-DNA

211 code has been shown to provide accurate dose point kernels for incident electron energies

212 ranging from $10 \mathrm{keV}$ to $100 \mathrm{keV}$.

213

214 Acknowledgements

215 The Geant4-DNA project received funding partly from the French Agence Nationale de la

216 Recherche under contract number ANR-09-BLAN-0135-01 and from the European Space

217 Agency under contract number AO6041- 22712/09/NL/AT. This work has been developed as 218 part of the activities planned in the project PICS 5921 (THEOS) of the Centre National de la 219 Recherche Scientifique. 


\section{Figure 1:}

221 (Color online) Comparison between the scaled dose point kernel distributions obtained by the

222 different numerical track-structure codes studied in the present work: GEANT4-DNA (red),

223 EGSnrc (green), PENELOPE (blue), CPA100 (cyan), MCNPX (magenta) and FLUKA
224 (orange). Panel
a) $E_{0}=10 \mathrm{keV}$. Panel
b) $E_{0}=30 \mathrm{keV}$. Panel
c) $E_{0}=50 \mathrm{keV}$.

225 Panel d) $E_{0}=100 \mathrm{keV}$.

\section{6}
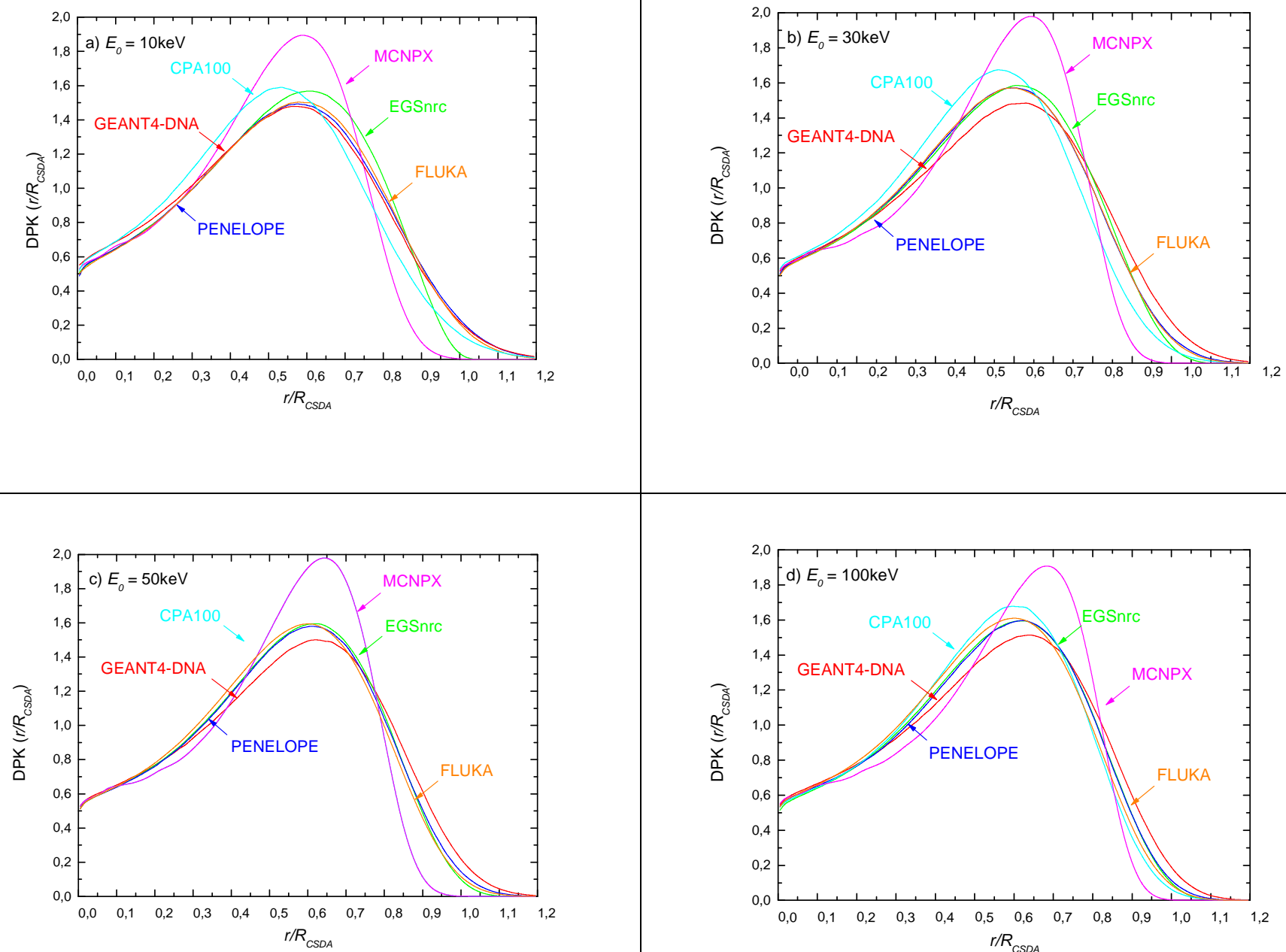
228 Table 1:

229 Comparison between the continuous-slowing-down-approximation range $R_{C S D A}(\mu \mathrm{m})$ obtained 230 by the different numerical track-structure codes studied in the present work.

\begin{tabular}{|c|c|c|c|}
\hline $\boldsymbol{E}_{\boldsymbol{0}}$ & $\begin{array}{c}\boldsymbol{R}_{\text {CSDA }} \\
\text { (GEANT4-DNA) }\end{array}$ & $\begin{array}{c}\boldsymbol{R}_{\text {CSDA }} \\
\text { (PENELOPE) }\end{array}$ & $\begin{array}{c}\boldsymbol{R}_{\text {CSDA }}{ }^{*} \\
\text { (EGSnrc, CPA100, KLUKA, MCNPX) }\end{array}$ \\
\hline $\mathbf{1 0} \mathbf{~ k e V}$ & 2.76 & 2.52 & 2.52 \\
\hline $\mathbf{3 0} \mathbf{~ k e V}$ & 18.16 & 17.57 & 43.20 \\
\hline $\mathbf{5 0} \mathbf{~ k e V}$ & 44.07 & 43.21 & 143.10 \\
\hline $\mathbf{1 0 0} \mathbf{~ k e V}$ & 144.12 & 143.06 & \\
\hline
\end{tabular}

231 "Note that the EGSnrc, CPA100, FLUKA and MCNPX values have been taken from the NIST

232 web database ESTAR [24] contrary to the other data here reported. 


\section{References}

235 [1] A.I. Kassis, Radiobiologic principles in radionuclide therapy, J. Nucl. Med. 46 (2005) $2364 \mathrm{~S} 12 \mathrm{~S}$.

237 [2] E.D. Yorke, L.E. Williams, A.J. Demidecki, D.B. Heidorn, P.L. Roberson, B.W. Wessels, 238 Multicellular dosimetry for beta-emitting radionuclides: autoradiography, thermoluminescent 239 dosimetry and three-dimensional dose calculations, Med. Phys. 20 (1993) 543-550.

240 [3] M.R. Puncher, P.J. Blower, Radionuclide targeting and dosimetry at the microscopic level: 241 the role of microautoradiography, Eur. J. Nucl. Med. 21 (1994) 1347-1365.

242 [4] F. Chehade, C. de Labriolle-Vaylet, N. Moins, M.F. Moreau, J. Papon, P. Labarre, 243 P. Galle, A. Veyre, E. Hindié, Secondary ion mass spectrometry as a tool for investigating 244 radiopharmaceutical distribution at the cellular level: the example of I-BZA and 14C-I-BZA, 245 J. Nucl. Med. 46 (2005) 1701-1706.

246 [5] W.E. Bolch, L.G. Bouchet, J.S. Robertson, B.W. Wessels, J. A. Siegel, R.W. Howell, 247 A.K. Erdi, B. Aydogan, S. Costes, E.E. Watson, A.B. Brill, N.D. Charkes, D.R. Fisher, M.T. 248 Hays, S.R. Thomas, MIRD Pamphlet No. 17: the dosimetry of nonuniform activity 249 distributions-radionuclide $\mathrm{S}$ values at the voxel level. Medical Internal Radiation Dose 250 Committee, J. Nucl. Med. 40 (1999) 11S-36S.

251 [6] L. Strigari, E. Menghi, M. d'Andrea, M. Benassi, Monte Carlo dose voxel kernel 252 calculations of beta-emitting and Auger-emitting radionuclides for internal dosimetry: a 253 comparison between EGSnrcMP and EGS4, Med. Phys. 33 (2006) 3383-3389.

254 [7] W.B. Li, W. Friedland, E. Pomplun, P. Jacob, H. Paretzke, M. Lassmann, C.H.R. Reiners, 255 Track structures and dose distributions from decays of 131I and 125I in and around water 256 spheres simulating micrometastases of differentiated thyroid cancer, Radiat. Res. 156 (2001) 257 419-429. 
258 [8] C. Champion, Theoretical cross sections for electron collisions in water: structure of 259 electron tracks, Phys. Med. Biol. 48 (2003) 2147-2168.

260 [9] C. Champion, A. L'hoir, M.F. Politis, P.D. Fainstein, R.D. Rivarola, A. Chetioui, A 261 Monte Carlo code for the simulation of heavy-ion tracks in water, Radiat. Res. 163 (2005) 262 222-231.

263 [10] C. Champion, C. Le Loirec, Positron follow-up in liquid water. I. A new Monte Carlo 264 track-structure code, Phys. Med. Biol. 5 (2006) 1707-1723.

265 [11] S. Uehara, H. Nikjoo, D.T. Goodhead, Comparison and assessment of electron cross 266 sections for Monte Carlo track structure codes, Radiat. Res. 152 (1999) 202-13.

267 [12] I. Kawrakow, Accurate condensed history Monte Carlo simulation of electron transport :

268 I. EGSnrc, the new EGS4 version, Med. Phys. 27 485-98 (2000).

269 [13] S. Salvat, J.M. Fernandez-Varea, J. Sempau, PENELOPE-2006, A Code System for 270 Monte Carlo Simulation of Electron and Photon Transport, OECD ISBN 92-64-02301-1 271 (2006).

272 [14] M. Terrissol and A. Baudre, A simulation of space and time evolution of radiolytic 273 species induced by electrons in water, Radiat. Prot. Dosim. 31 (1990) 175-177.

274 [15] FLUKA: A Multi-Particle Transport Code. Geneva: CERN European organization for 275 nuclear research; 2005.

276 [16] MCNPX User's Manual, Version 2.5.0, Laurie Waters, ed., LA-CP-05-0369 (2005).

277 http://mcnpx.lanl.gov/documents.html.

278 [17] S. Incerti, A. Ivanchenko, M. Karamitros, A. Mantero, P. Moretto, H.N. Tran, B. 279 Mascialino, C. Champion, V.N. Ivanchenko, M.A. Bernal, Z. Francis, C. Villagrasa, G. 280 Baldacchino, P. Guèye, R. Capra, P. Nieminen, C. Zacharatou, Comparison of GEANT4 very 281 low energy cross section models with experimental data in water, Med. Phys. 37 (2010) 46922824708. 
283 [18] I. Kawrakow and A.F. Bielajew, On the representation of electron multiple elastic-

284 scattering distributions for Monte Carlo calculations, Nucl. Instrum. Methods Phys. Res. B 285134 (1998) 325-35.

286 [19] E. Mainegra-Hing, D.W.O. Rogers, I. Kawrakow, Calculation of photon energy 287 deposition kernels and electron dose point kernels in water, Med. Phys. 32 (2005) 685-699.

288 [20] F. Salvat, J. Sempau, and J.M. Fernandez-Varea, Tech. Rep., Universitat de Barcelona, 2892006.

290 [21] F. Botta, A. Mairani, G. Battistoni, M. Cremonesi, A. Di Dia, A. Fassò, A. Ferrari, M.

291 Ferrari, G. Paganelli, G. Pedroli, M. Valente, Calculation of electron and isotopes dose point 292 kernels with FLUKA Monte Carlo code for dosimetry in nuclear medicine therapy, Med. 293 Phys. 38 (2011) 3944-3954.

294 [22] D.R. Schaart, J.T.M Jansen, J. Zoetelief, P. De Leege, A comparison of MCNP4C 295 electron transport with ITS 3.0 and experiment at energies between $100 \mathrm{keV}$ and $20 \mathrm{MeV}$ :

296 Influence of voxel size, substeps and energy indexing algorithm, Phys. Med. Biol. 47 (2002), 297 1459-1484.

298 [23] H. Koivunoro, T. Siiskonen, P. Kotiluoto, I. Auterinen, E. Hippeläinen, S. Savolainen, 299 Accuracy of the electron transport in MCNP5 and its suitability for ionization chamber 300 response simulations: A comparison with the EGSNRC and PENELOPE codes, Med. Phys. 30139 (2012) 1335-1343.

302 [24] ESTAR, National Institute of Standards and Technology, «Stoping powers and Range 303 table for electrons », http://physics.nist.gov/PhysRefData/Star/Text/ESTAR.html.

304 [25] ICRU, «Stopping powers for electrons and positrons », ICRU Report 37, ICRU, 305 Washington, DC, 1984. 